\title{
Study on the Effect of the Rheological Properties of Melt on the Die Cross-Section Shape for the Plastic Tube Extrusion Forming
} \author{
Zhong REN ${ }^{1,2, a^{*}}$ and Xing-Yuan HUANG $^{2, b}$ \\ ${ }^{1}$ Key Laboratory of Optic-Electronic and Communication, Jiangxi Science and Technology Normal \\ University, Nanchang, 330038, China \\ ${ }^{2}$ School of Mechanical and Electrical Engineering, Nanchang University, Nanchang, 330031, China \\ arenzhong0921@163.com, b25017051@qq.com \\ ${ }^{*}$ Corresponding author
}

Keywords: Extrusion forming, Extrusion die design, Plastic tube, Extrudate swell, Numerical simulation.

\begin{abstract}
The die optimization design is an effective way of overcoming the extrusion problems of plastic tube. Due to the obvious influence of the rheological properties of melt on the extrusion shape of the plastic tube, the reasonable cross-section shape of the die is one of most important factors in the die design. In this study, the effects of rheological properties of polymer melt on the cross-section shape of plastic tube were investigated. Numerical results indicate that the inner radius and the outer radius of the die's cross-section shape increase with the increasing of the viscosity, and the parameter of $\varepsilon$ and $\xi$, but decrease with the increasing of the relaxation time. For the gap thickness of the die, the gas thickness increases with the viscosity, and the parameter of $\varepsilon$ and $\xi$, but decreases with the increasing of the melt's viscosity.
\end{abstract}

\section{Introduction}

The extrusion forming [1] is one of the most important methods of producing the plastic tube in the process of the plastic products. The plastic tube with different cross-section types can be obtained by using the method of the molten polymer extruded from a metal die with a fixed cross-section type. However, due to the properties of elastic energy recovery for the melt, the triggering of the high shear stress and shear rate in the flow channel, the plastic extrudates are easy to generate the some unstable phenomena, such as extrudate swell [2], extrudate fracture [3], and extrudate distortion [4]. Up to now, many methods have been studied to overcome these above mentioned problems, such as the fluoride additives [5], die surface modification [6], process optimization [7], and die design optimization [8]. where the method of die design optimization has the advantages of low price, high efficiency and short development time etc. However, there are some problems should be solved for the die design of the plastic tube extrusion forming, such as the effect of the physical properties for the melt, the effect of the process parameter, the effect of the die length, etc. In this study, the effect of the rheological properties of the melt on the reverse design of the plastic tube extrusion forming was numerically investigated by using the computed fluid dynamics software POLYFLOW [9]. This study has an important guidance value in the die reverse design of the plastic tube with different physical properties.

\section{Numerical Simulation}

Numerical Model. The geometric model of the die for the plastic tube extrusion forming is shown in Fig.1(a). 

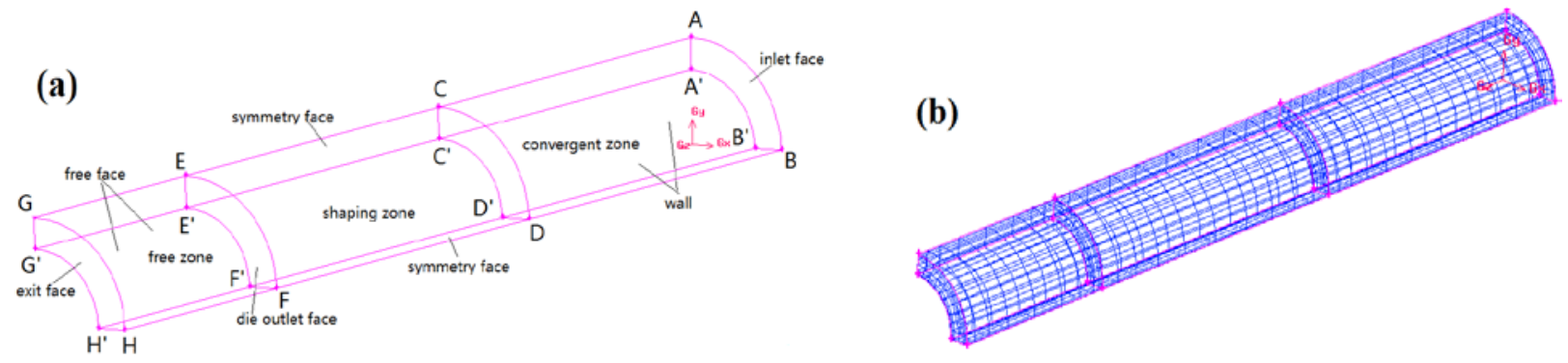

Fig.1 Geometric model of the die for the plastic tube extrusion forming

In Fig.1 (a), the 1/4 part structure was used due to the axis-symmetry property of the plastic tube. The section of ABDC-A'B' D'C' is the convergent zone of the die, the section of CDFE-C'D'F'E' is the shaping zone of the die, the section of EFHG-E'F' $H^{\prime} G^{\prime}$ is the free zone of the die. The length of the convergent zone is $50 \mathrm{~mm}$, the length of the shaping zone is $50 \mathrm{~mm}$, the length of the free zone is $30 \mathrm{~mm}$, the thickness of the plastic tube is $3 \mathrm{~mm}$, the inner radius of the tube is $7 \mathrm{~mm}$, outer radius of the tube is $10 \mathrm{~mm}$. The finite element mesh of the Fig.1(a) is shown in Fig.1(b). In Fig.1(b), the meshes are properly refined near the inlet face, the inlet face of the shaping zone, die outlet face, exit face, the inner wall, and outer wall of the tube. The mesh number of the Fig.1(b) is 1800.

Hypotheses. In order to conveniently study the effect of rheological properties of melt on the die design of the plastic tube, some reasonable hypotheses should be set as follows,

1) The melt is regarded as the isothermal, incompressible, steady, laminar Non-Newtonian fluid.

2) The inertia force, gravity force and thermal irradiation effect of the melt are neglected.

Governing Equations. According to the above mentioned hypotheses, the mass and momentum conservation equations are given as follows,

$$
\begin{gathered}
\nabla \cdot v=0 \\
\nabla p-\nabla \cdot \tau=0
\end{gathered}
$$

where $\nabla$ Hamilton operator, $v$ is the velocity vector of the melt, $p$ is the pressure, $\tau$ is the extra stress of the melt.

In this study, Phan-Thien and Tanner(PTT) constructive equation [10] was used as the constructive equation of polymer flow in the micro-channel due to the wide range of shear rate. PTT constructive equation can be shown as follows,

$$
\begin{gathered}
\tau=\tau_{1}+\tau_{2} \\
\exp \left[\frac{\varepsilon \lambda}{\left(1-\eta_{r}\right) \eta} \operatorname{tr}\left(\tau_{1}\right)\right] \tau_{1}+\lambda\left[\left(1-\frac{\xi}{2}\right) \tau_{1}+\frac{\xi}{2} \iota_{1}\right] \\
=2\left(1-\eta_{r}\right) \eta D
\end{gathered}
$$

where $\tau$ is the total extra-stress tensor of the melt, $\tau_{1}$ and $\tau_{2}$ are the melt viscoelastic component and purely-viscous component of the total extra-stress tensor $(\tau)$, respectively. $\eta_{r}=\eta_{2} / \eta$ is the viscosity ratio, $\lambda$ is a model-specific relaxation time, $\xi$ and $\varepsilon$ are material properties controlling the shear viscosity and elongational behavior of polymer melt, respectively. $D$ is the melt rate-of-deformation tensor. $\stackrel{\nabla}{1}_{1}$ and ${ }_{\tau} \tau_{1}$ is the upper-convected and below-convected time derivative of the viscoelastic extra stress tensor $(\tau)$, respectively

Boundary Conditions. According to the Fig.1(a), the boundary conditions are set as follows,

1) Inlet face: Supposing that the flow of the melt in the tube is the full-developed, steady and laminar flow, the following relationships should be satisfied, i.e., $\partial v_{z} / \partial z=0, v_{x}=v_{y}=0$. where 
$v_{x}, v_{y}$ and $v_{z}$ are the velocities of melt at the direction of $x, y$ and $z$ coordinate. The volume flow rate of $0.1 \mathrm{~cm}^{3} / \mathrm{s}$ was imposed on the inlet face.

2) Wall: For the traditional extrusion forming, no-slip boundary condition was imposed on all walls, i.e., $v_{n}=0, v_{s}=0$.

3) Symmetric face: The following conditions should be satisfied, i.e., $f_{s}=0, v_{n}=0$.

4) Free face: for the outer surface of the tube, the following conditions should be satisfied, i.e., $f_{n}=0, f_{s}=0$. However, for the inner surface of the tube, the normal force should be imposed because the gas with a certain of pressure is often imposed in the inner lumen in order to avoid the cavity adhesion of the tube. That is, $f_{n}>0, f_{s}=0$. In the simulations, $f_{n}=100 P a$.

5) Exit face: Supposing the traction forces was imposed on the exit face, that is, the normal stress are not equal to zero, i.e., $f_{n}>0$ and $v_{s}=0$.In the simulations, $f_{n}=100 \mathrm{~Pa}$.

Material Parameters. For the numerical simulation of the molten plastic tube extrusion forming, the material parameters are shown in Table 1.

Table 1 Material parameters of the plastic tube

\begin{tabular}{cccccc}
\hline Parameters & $\eta$ (Pa.s) & $\lambda(\mathrm{s})$ & $\varepsilon$ & $\xi$ & $\eta_{r}$ \\
\hline Value & 2700 & 0.2 & 0.18 & 0.23 & 0.12 \\
\hline
\end{tabular}

\section{Numerical Results and Analyses}

Reverse Extrusion Simulation Result. The reverse extrusion prediction result of the plastic tube with the material parameters given in Table 1 was obtained, which is shown in Fig.2.

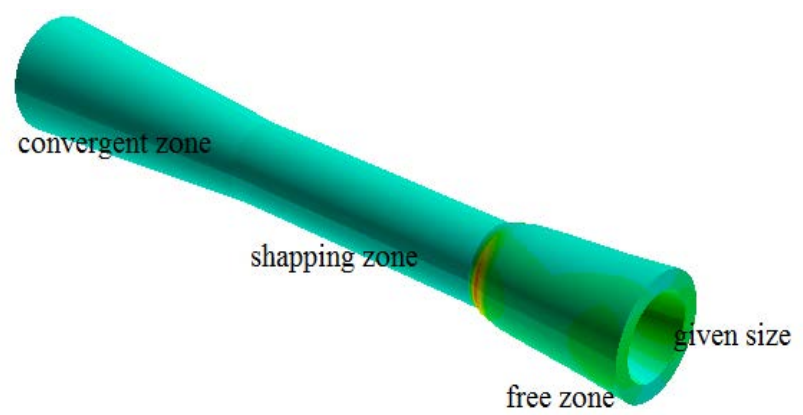

Fig.2 Reverse extrusion simulation result of plastic tube

From Fig.2, it can be seen that the outlet size of the die shaping section is less than that of the given size of the exit face for a plastic tube, which can be explained by the effect of the elastic energy storage recovery when the molten polymer is extruded from the die channel. In addition, it can be seen that the convergent profile with a certain angle is generated in the convergent zone.

Effect of the Viscosity on the Die Outlet Cross-Section Shape. In the simulations, to study the effect of the viscosity for the melt on the die outlet cross-section shape, four different viscosities were used, that is, 1350, 2700, 5400, and 8100(Pa.s). The inner radius and outer radius changes of the plastic tube with the increasing of the viscosity for the melt are shown in Fig.3. 


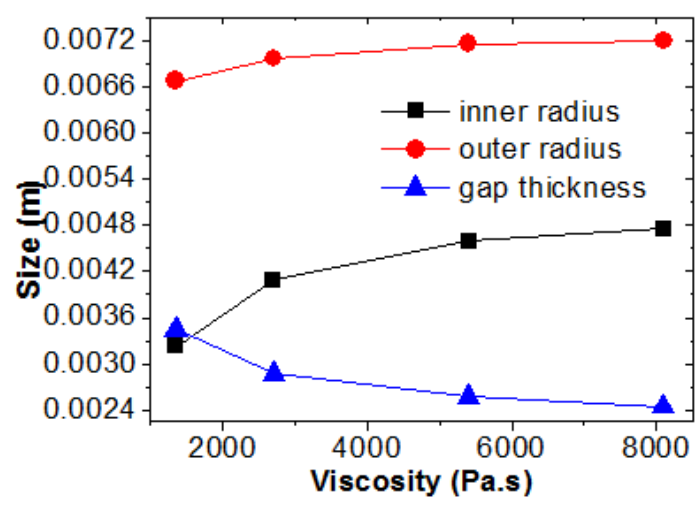

Fig.3Effect of the viscosity of melt on the die outlet cross-section shape

From Fig.3, it can be seen that the inner and outer radius of the plastic tube all increase with the increasing of the viscosity for the melt. Moreover, the increasing degree of inner radius is larger than that of the outer radius. In addition, the gap thickness of the die shaping zone decreases with the increasing of the viscosity because the elastic property of the melt increases with the increasing of the viscosity.

Effect of the Relaxation Time on the Outlet Cross-Section Shape of the Die. To study the relaxation time of the melt on the outlet cross-section shape of the die, four different relaxation times of the melt were used. The effects of the relaxation time on the outlet cross-section shape of the die are shown in Fig.4.

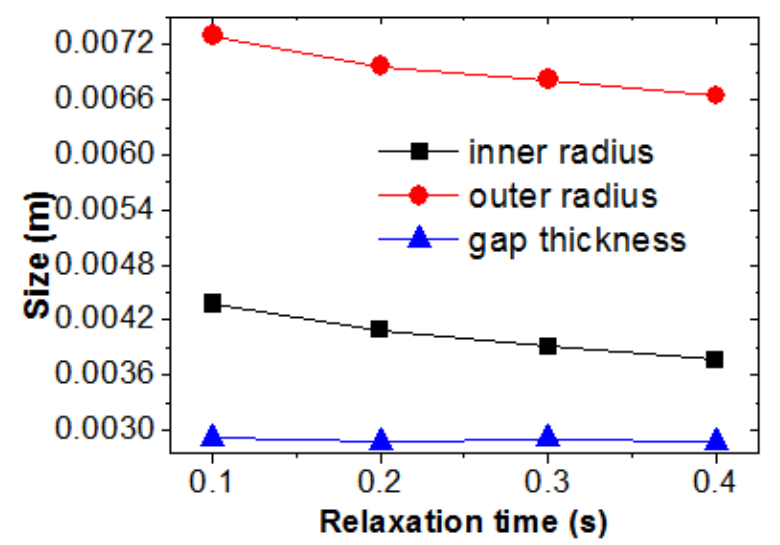

Fig.4 Effects of the relaxation time on the outlet cross-section shape of the die

From Fig.4, it can be seen that the inner and outer radius of the die shaping zone decreases with the increasing of the relaxation time of the melt because the elastic energy storage and extrudate swell effect of the melt increase with the increasing of the relaxation time of the melt. However, it can be also seen that the gap thickness does nearly not change, which demonstrates that the gas thickness of the die is not impacted by the relaxation time of the melt.

Effects of the Parameter of $\varepsilon$ and $\xi$ on the Outlet Cross-Section Shape of the Die. To study the effects of the parameter of $\varepsilon$ and $\xi$ on the outlet cross-section shape of the die for the plastic tube extrusion forming, four different parameters of $\varepsilon$ and $\xi$ were used, that is, $\varepsilon=0.1,0.23,0.4,0.6$, and $\xi=0.1,0.18,0.4,0.6$. The effect results are shown in Fig.5. 

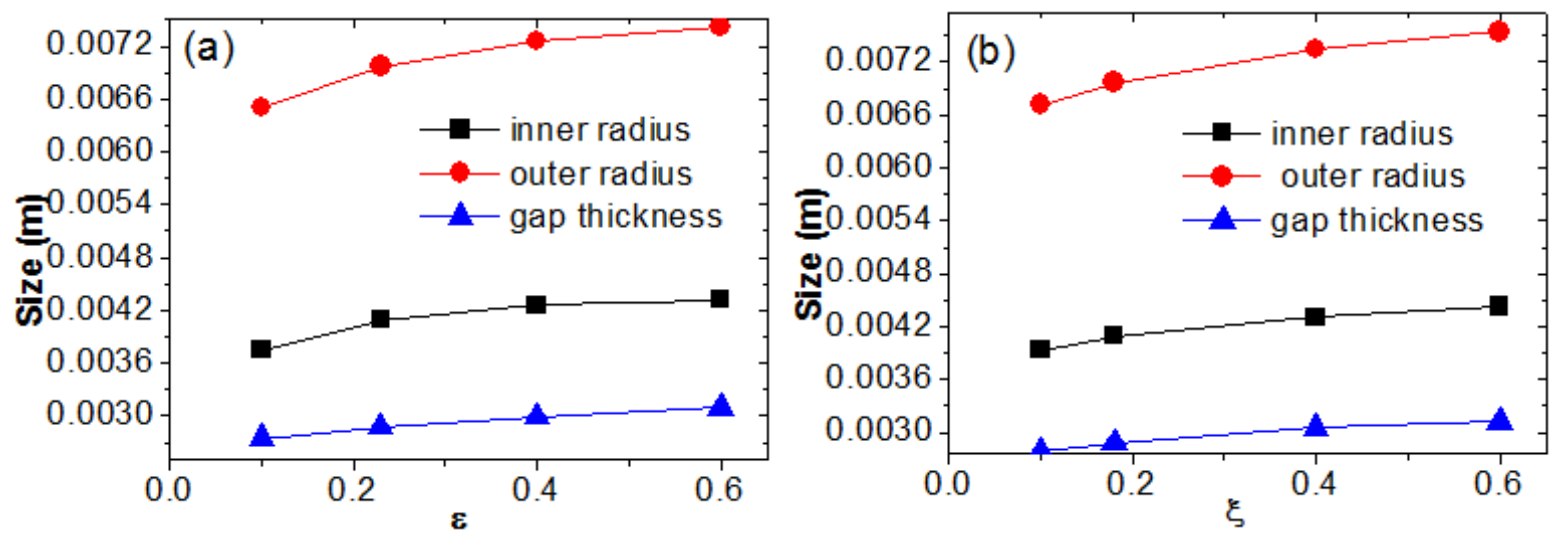

Fig.5 Effect of the parameter of $\varepsilon$ and $\xi$ on the outlet cross-section shape of the die

From Fig.5, it can be seen that the inner radius, outer radius and gap thickness of the cross-section shape of the die for the plastic tube increase with the increasing of the parameter of $\varepsilon$ and $\xi$, which demonstrates that the viscous and elastic properties of the polymer melt decreases with the increasing of the parameter of $\varepsilon$ and $\xi$.

\section{Summary}

The plastic tube is a kind of important products widely used into many fields, such as biomedical diagnosis, architectural engineering, and chemical engineering. Extrusion forming is a popular processing method of producing the plastic tube. However, the plastic tube is usually occurred some problems, such as, extrudate swell, melt fracture, and extrudate distortion. The die optimization design is a kind of effective way to avoid these above mentioned problems. In this study, the effects of rheological properties of polymer melt on the cross-section shape of plastic tube were investigated. Numerical results indicate that the inner radius and the outer radius of the die's cross-section shape increase with the increasing of the viscosity, and the parameter of $\varepsilon$ and $\xi$, but decrease with the increasing of the relaxation time. For the gap thickness of the die, the gas thickness increases with the viscosity, and the parameter of $\varepsilon$ and $\xi$, but decreases with the increasing of the melt's viscosity.

\section{Acknowledgements}

This work is supported by the Natural Science Foundation of Jiangxi Province (20151BAB202011), the Science and Technology Pillar Program of Jiangxi Province (20132BBG70103), Top-notch talent foundation project of JXSTNU (2014QNBJRC004), the Scientific Research Foundation of Jiangxi Provincial Education Bureau (No. GJJ150814), and 2011 Cooperative Innovation Center Project of Jiangxi Province (2014XTCX005).

\section{References}

[1] W. Krzysztof, A method for estimation of polymer melt temperature fluctuation in a single screw extrusion process. Polym. Eng. Sci. 3 (2001) 235-241

[2] Y. Otsuki, T. Kajiwara, K. Funatsu, Numerical simulations of annular extrudate swell using various types of viscoelastic models. Polym. Eng. Sci. 39 (1999) 1969-1981

[3] C. Tzoganakis, B.C. Price, S.G. Hatzikiriakos, Fractal analysis of the sharkskin phenomenon in polymer melt extrusion. J. Rheol. 37 (1993) 355-366

[4] D.S. Kalika, M.M. Denn, Wall slip and extrudate distortion in linear low-density polyethylene, J. Rheol. 8 (1987) 815-834 
[5] R.D. Arda, M.R. Mackley, The effect of die exit curvature, die surface roughness and a fluoropolymer additive on sharkskin extrusion instabilities in polyethylene processing. J. Non-Newtonian Fluid Mech.126 (2005) 47-61

[6] J.M. Piau, N. El Kissi, F. Tousssaint, A. Mezghant, Distortions of polymer melt extrudates and their elimination using slippery surfaces. Rheol. Acta 34 (1995) 40-57

[7] Z. Jurkovič, M. Brezočnik, B. Grizelj, V. Mandič, Optimization of extrusion process by genetic algorithms and conventional techniques. Tehnicki Vjesnik 16 (2009) 27-33

[8] A. Sadollah, A. Bahreininejad, Optimization of die design using metaheuristic methods in cold forward extrusion process. Neural Comput. Appl. 21 (2012) 2071-2076

[9] ANSYS Inc. ANSYS POLYFLOW 14.5 User’s Guide, ANSYS. Inc., Southpointe, 2012

[10] N.P. Thien, R.I. Tanner, A new constitutive equation derived from network theory. J. Non-Newton Fluid. Mech. 2 (1977) 353-365 